\title{
To reduce the average length of stay of patients who are admitted for DA- EPOCH-R chemotherapy regimen
}

Yee Mei Lee

National Cancer Institute, Singapore

\begin{abstract}
Healthcare institutions are often faced with bed crunch situation. As a result, patients requiring inpatient hospital stay for cancer treatment are delayed and this could lead to compromised overall disease response. Apart from the early discharge of patients to step-down care and explore alternatives of treatment setting, one of the ways to reduce length of stay is to improve on the efficiency of work processes.

A baseline study demonstrated that delays in prescribing chemotherapy orders has led to an increased inpatient stay of seven days for a five day treatment regimen. This has profound consequences in terms of costs, patient safety, and utilisation of healthcare resources. A quality improvement project was initiated to review and revise the workflow and processes involved for the entire episode of treatment. A postimplementation review of the interventions showed cost savings, a reduction of average length of stay from seven days to six days (with a total of 28 days saved over six months), and improved patient and staff experience.
\end{abstract}

\section{Problem}

Delayed in prescribing chemotherapy orders resulted in delayed drug preparation and administration of treatment. This has led to increased length of hospital stay, patient dissatisfaction, and suboptimal utlisation of healthcare resources.

\section{Background}

Dose adjusted EPOCH plus rituximab (DA-EPOCH -R) is an effective treatment regimen against a variety of lymphoma with histological subtypes such as diffuse large B-cell, primary mediastinal (thymic) large B-cell and others.[1] This regimen requires patients to be hospitalised as it is vital that there is no interval in between drug administrations, and four out of the five days of the treatment includes concurrent continuous infusion of three cytotoxic drugs.

The regimen is associated with the following side effects: mild to moderate emetogenicity, constipation, hyperglyceamia, alopecia, and bone marrow suppression.[2] In addition, patients are required to learn to administer subcutaneous injection granulocyte colonystimulating factor (GCSF) beginning on day six and continue until the absolute neutrophil count (ANC) is more than $5 \times 10(9)] / l$.

Patients are also provided with education on self-care and central venous catheter management before they are discharged home. However, the intricacy of the chemotherapy regimen (in addition to the time needed to educate and prepare patients and caregivers before discharge) has inadvertently resulted in increased length of hospital stay.

In the recent years, there have been increasing efforts to transfer care to ambulatory settings in the institution, partly to address the issue of bed crunch. Where care has been transferred to ambulatory settings, there were documented benefits such as reduced risk of hospital acquired infection and functional decline.[3] For cancer patients, it has shown to improve health and quality of life for those patients who prefer to spend more time in the comfort of their own home while undergoing treatment.[4] However, the administration of DA-EPOCH-R as an outpatient regimen was not an option in this institution because there was no portable infusion pump available to accommodate a large amount of infusate. The other barrier was related to the way payment and reimbursement structure of healthcare expenses. Patients were faced with a higher out-of-pocket cash payment for the total cost should they opt for treatment at the ambulatory care setting.

\section{Baseline measurement}

For this project, the following baseline measurements were included. Adult patients who were scheduled for treatment every 21 days were monitored over a period of three months for:

a. Length of hospital stay

b. Time of chemotherapy orders were prescribed

c. Time of arrival of chemotherapy drugs at the inpatient units

d. Time of commencement of chemotherapy

e. Time of completion of drugs administered to time of discharge.

Overall, data for a total of 20 admissions to the hospital for chemotherapy were captured. The length of stay ranged between seven to ten days especially for those who received treatment for the first time. Doctors took more than 12 hours (from the time of patient's admission to the ward) before the chemotherapy orders were prescribed. This is because patients are often admitted the 
evening before the actual day of treatment due to limited bed availability.

Chemotherapy orders were only prescribed on the morning of treatment after the morning ward round is completed. From the time the prescription was completed to the drugs delivery to inpatient units, the duration taken was approximately four hours of waiting time.

Even after the drugs arrived at the inpatient unit, it took about one to two hours before treatment was started. This was due to other competing nursing activities taking place at the time. Once treatment was completed, patients spent another 10 to 12 hours (from the time of completion of treatment to the time of discharged) before they were discharged from the hospital. The delay is caused by treatment which ends at late at night and arrangement for discharge was usually done during work hours the following day.

See supplementary file: ds5103.pdf - "Baseline measurement and problem analysis"

\section{Design}

A focus group was conducted among physicians, nurses, clinic assistants, and pharmacists to determine the underlying issues. Separates sessions were held to brainstorm for solutions. Based on the gaps identified, the team decided to work on changing some old practices. First was the decision to engage the physicians to order the necessary laboratory tests during clinic consultation session a few days prior to scheduled admission. Blood results that fall within normal ranges are valid within a week for chemotherapy orders unless patients become unwell in between. Chemotherapy prescribing orders can then be completed by the primary physician at least 24 hours before the scheduled admission date to the hospital.

Second was to collaborate with the clinic assistants and pharmacists to ensure the chemotherapy orders were transmitted timely to the satellite pharmacy. This facilitated the workflow for pharmacists to prepare the necessary documents prior to the actual treatment day.

Thirdly was to work with the porters to streamline their workflow for drug delivery to the inpatient unit and to minimise batching that caused further delays.

Another important stakeholder was the nurses at the inpatient setting. Nurses at all levels were engaged to work on setting priorities for timely chemotherapy administration and agreement was sought to review current internal workflow. Early commencement of patient education and initiation of discharge planning were strongly emphasised in order to achieve smooth transition of care for patients from hospital to home.

A standardised discharge plan with specific instructions for frequency of blood checks and clinic consultations was also implemented to ease the work flow for nurses and administrative staff.

\section{Strategy}

At the planning stage, there were several issues in the workflow that needed change in practice and work group members had to ensure the implementation would not be hindered due to lack of staff awareness, frequent rotation of staff, shortage of manpower, and unfamiliarity of the new workflow. The aims were to reduce patients' length of stay from seven to at least six days through interventions targeted at the several levels.

PDSA cycle 1: Posters in the clinic rooms were used to remind doctors to write up chemotherapy orders. Clinic assistants were in charge of printing out chemotherapy order forms and making them available in the consult rooms. Pharmacists were informed of a patient's planned admission and would receive a fax copy of chemotherapy orders. Porters were reinforced on timeliness in drug delivery while inpatient nurses were tasked to follow up on chemotherapy orders and commence chemotherapy as soon as drugs arrive in the ward. Medical team and nursing team are reminded to initiate patient education and discharge management on the day of admission.

About a month into the implementation of the new work flow, the preliminary analysis showed favourable results. Length of stay was reduced from seven to six days. Patients were able to go home immediately upon completion of treatment as the discharge medications and follow up dates were already pre-prepared. Physicians were able to prescribe the chemotherapy orders on time as the laboratory results were available.

PDSA cycle 2: Preliminary assessment of interventions implemented, there were several incidents whereby physicians had forgotten write up the chemotherapy orders before admission and it caused delays for some patients. To address this issue, posters were put up in the clinic rooms as reminders for doctors to write up chemotherapy orders. A simple chart was also developed for nurses and clinic assistants for reference when they are uncertain with the discharge management.

PDSA cycle 3: A new group of nurses joined the inpatient wards and there were a few incidents of not confirming chemotherapy orders early morning, instead showing that they were done only after the ward rounds which caused delays to the preparation of drugs. Ward nursing officers in charge were reminded to brief the new nurses and a reminder sheet was clipped together with the drugs when they were delivered from the pharmacy.

\section{Results}

The effects of a changed workflow and standardisation of discharge plan were monitored and the following results were seen. There was a reduction in average length of stay from seven to six days, and total days saved of 28 days over a period of six months. In terms of bed day charges, patients saved SGD 1000 per single episode of admission and SGD 6000 for six episodes of admissions. 


\section{BMJ Quality Improvement Reports}

Patients provided good feedback for the change initiated as it helped them to have more time with their family and cost savings. From the staff perspectives, they were in favour of this initiative as it eased the bed crunch situation and improved patient safety (see attachment).

See supplementary file: ds5104.pdf - "Results and Feedback"

\section{Lessons and limitations}

The effects of change in the workflow have brought about several positive outcomes in addition to those measured. Pharmacists are able to plan ahead and prioritise preparation (avoiding peak hours) when chemotherapy orders are received at least one day prior to admission. It was observed that nurses become more efficient in their work and chemotherapy administration, ensuring that there are fewer competing patient-care activities in the ward when the drugs arrive at the inpatient unit.

In terms of patient safety, it was reported anecdotally that there was a reduced risk in patient safety when chemotherapy commences on the day when more doctors are available in the ward. Overall, this is more cost effective for patients and improves efficiency in the utilisation of healthcare resources.

There were also challenges faced by the work group members. They include constant reminders for physicians to write out the chemotherapy orders before patients are admitted for treatment, along with the coordination of each personnel's responsibilities involved in the entire process (especially when they have other roles to fulfill). The most important lesson learnt from this project was that effective communication at all levels was key in making this improvement project a success.

\section{Conclusion}

A small effort with an interdisciplinary team collaboration made a difference in patients' overall experience and improves in the efficiency of human resource and bed utilisation.

Teamwork, communication, and involvement of all stakeholders play an important role for this project to be successful and sustainable in the long run.

\section{References}

1. Dunleavy K, Pittaluga S, Maeda LS, et al. Dose adjusted EPOCH-rituximab therapy in primary mediastinal B-cell lymphoma. N Engl J Med 2013; 368:1408-16.

2. Wilson WH, Grossbard, ML, Pittaluga S, et al. Doseadjusted EPOCH chemotherapy for untreated large B-cell lymphomas: a pharmacodynamic approach with high efficacy. Blood 2002; 99(8):2685-93.

3. Alexander JG, Amelia KB, James ES, et al. Hospitalacquired infection underlies poorfunctional outcome in patients with prolonged length of stay. ISRN Stroke vol. 2013, Article ID 312348.
4. Rubenstein EB. Costs and benefits of outpatient therapy. Support Care Cancer 1994; 2(5):307-11.

\section{Declaration of interests}

No funding or conflict of interest to declare.

\section{Acknowledgements}

I wish to thank all the staff at the cancer centre and inpatient ward nurses who have helped in data collection for the project.

\section{Ethical approval}

The project was exempted from ethical approval because it is an improvement study. 